\title{
Transcriptome analysis reveals dysregulated long non-coding RNAs and mRNAs associated with extrahepatic cholangiocarcinoma progression
}

\author{
FUMIN ZHANG $^{1-3^{*}}$, MING WAN $^{1 *}$, YI XU ${ }^{1}$, ZHENGLONG LI $^{1}$, PENGCHENG KANG $^{1}$, XINGMING JIANG $^{1}$, \\ YIMIN WANG ${ }^{1}$, ZHIDONG WANG ${ }^{1}$, XIANGYU ZHONG ${ }^{1}, \mathrm{CHUNLONG} \mathrm{LI}^{1}$ and YUNFU CUI ${ }^{1}$ \\ ${ }^{1}$ Department of Hepatopancreatobiliary Surgery, Second Affiliated Hospital of Harbin \\ Medical University, Harbin, Heilongjiang 150000; ${ }^{2}$ Department of General Surgery, Daqing Oilfield \\ General Hospital, Daqing, Heilongjiang 163001; ${ }^{3}$ The Key Laboratory of Myocardial Ischemia, \\ Harbin Medical University, Ministry of Education, Harbin, Heilongjiang 150086, P.R. China
}

Received February 26, 2016; Accepted February 7, 2017

DOI: $10.3892 / 01.2017 .6987$

\begin{abstract}
The incidence of extrahepatic cholangiocarcinoma (ECC) is the highest of all the cholangiocarcinoma cases. However, the molecular mechanism of ECC genesis and progression remains unclear. Long non-coding RNAs (lncRNAs) have been revealed to perform critical regulatory roles in cancer biology. In order to understand lncRNA expression patterns and their potential function in ECC, a transcriptome analysis of 1ncRNA and mRNA expression was performed in ECC and paired adjacent non-cancerous tissues using Agilent human lncRNA + mRNA arrayV4.0 (4x180 K format). It was identified that 268 lncRNAs and 459 mRNAs were differentially expressed in ECC. Among these, 78 lncRNAs and 66 mRNAs were upregulated $>2$-fold compared with adjacent non-cancerous tissues, and 190 lncRNAs and 393 mRNAs were downregulated in the ECC samples. Differences in lncRNA expression between ECC and paired adjacent non-cancerous tissues were confirmed using reverse transcription-quantitative polymerase chain reactionas proof of principle. Functional analysis of co-expressed mRNAs with lncRNAs indicated that these dysregulated lncRNAsmay be involved in known ECC-associated biological processes and pathways. The present findings indicated that mRNAs and lncRNAs perform important roles in the development and progression of ECC.
\end{abstract}

Correspondence to: Professor Yunfu Cui, Department of Hepatopancreatobiliary Surgery, Second Affiliated Hospital of Harbin Medical University, 246 Xuefu Road, Harbin, Heilongjiang 150000, P.R. China

E-mail: yfcui777@hotmail.com

*Contributed equally

Key words: extrahepatic cholangiocarcinoma, gene expression, long non-coding RNAs, microarray
The present findings may lay the foundation for future efforts to understand the role of lncRNAs and develop novel biomarkers in ECC

\section{Introduction}

Extrahepatic cholangiocarcinoma (ECC) is a highly malignant cancer, representing $\sim 80 \%$ of all cholangiocarcinoma clinical cases. In previous years, the incidence and mortality of ECC has continued to increase worldwide (1). Although continued advances in surgical techniques and treatment strategies have been achieved, the 5-year survival rate for patients who undergo surgical resection has been reported to be only $20-40 \%(2,3)$. The main reasons for the poor prognosis of ECC are a low rate of early diagnosis, fast progression and a high rate of recurrence. There is currently no effective method to improve the diagnosis and treatment of ECC, and the major cause of the molecular pathogenesis of oncogenesis and the progression of ECC remains largely unclear.

Long non-coding RNAs (lncRNAs) are a large class of ncRNAs. It has been revealed that IncRNAs are involved in the development and progression of tumors, and that their abnormal expression is associated with tumor proliferation, apoptosis, the cell cycle, angiogenesis, recurrence and metastasis in numerous different types of cancer (4,5). Previous studies have demonstrated the potential roles of lncRNAs to serve as diagnostic markers and therapeutic targets for cancers (6-14). However, the role and mechanism of lncRNAs in ECC remains largely unknown. Via transcriptome analysis, the present study aimed to investigate lncRNA and mRNA expression that is up- or downregulated in ECC tissues compared with paired peritumoral tissues. Additional bioinformatics analysis and validation studies were performed to reveal an association between clinical characteristics and lncRNA expression levels. These analyses and observations indicated that alterations in IncRNA expression may become a novel biomarker or therapeutic target for ECC diagnosis and treatment. 


\section{Materials and methods}

Patients and tissue samples. A total of 42 patients with ECC who underwent surgical resection at the Second Affiliated Hospital of Harbin Medical University (between January 2013 and October 2015) were included in the present study. All patients provided their written informed consent for inclusion in this study prior to surgery. Patients who were treated with preoperative radiotherapy or chemotherapy were excluded. ECC tissues and paired adjacent non-cancerous tissues were collected and immediately frozen in liquid nitrogen. Matched non-cancerous tissues were obtained from regions of at least $3 \mathrm{~cm}$ distant from the tumor borders (China National Genebank v1.00). A total of 3 pairs of samples were used for microarray analysis, and all samples were subjected to reverse transcription-quantitative polymerase chain reaction (RT-qPCR).

lncRNA and mRNA microarrays. The Agilent human lncRNA + mRNA Array v4.0 was designed with four identical arrays per slide (4x180 K format), with each array containing probes interrogating $\sim 41,000$ human lncRNAs and 34,000 human mRNAs (Agilent Technologies, Inc., Santa Clara, CA, USA). Those IncRNA and mRNA target sequences were mergedfrom multiple databases: 23,898 from GENCODE (http://www.gencodegenes.org/)/ENSEMBL(http://www.ensembl. org); 14,353 from Human LincRNA Catalog (15); 7,760 from RefSeq (https://www.ncbi.nlm.nih.gov/refseq/); 5,627 from UCSC (https://genome.ucsc.edu/); 13,701 from ncRNA Expression Database; 21,488 from LNCipedia; 1,038 from H-InvDB; 3,019 from lncRNAs-a (Enhancer-like); 1,053 from antisense ncRNA pipeline; and 407 Hox ncRNAs, 962 upstream conserved regions (UCRs) and 848 lncRNAs from the Chen Ruisheng lab (Institute of Biophysics, Chinese Academy of Science, Shanghai, China). Each RNA was detected by probes, and experiments were repeated twice. The array also contained 4,974 Agilent control probes (Agilent Technologies, Inc.).

RNA extraction and quality control. Total RNA was extracted from 42 pairs of frozen ECC tissues and matched non-cancerous tissues using TRIzol reagent (Thermo Fisher Scientific, Inc., Waltham, MA, USA) according to the manufacturer's protocol. Tissue $(50-100 \mathrm{mg}$ ) was homogenized with $1 \mathrm{ml}$ TRIzol reagent in a round-bottomed tube using a glass Teflon homogenizer, and the homogenized sample was incubated for $10 \mathrm{~min}$ at room temperature. The sample was then centrifuged at $12,000 \mathrm{xg}$ for $10 \mathrm{~min}$ at $4^{\circ} \mathrm{C}$ and the cleared supernatant was transferred to a new tube. Chloroform ( $0.3 \mathrm{ml}$ per $1 \mathrm{ml}$ TRIzol) was added to the tube, and the tube was agitated vigorously by hand for $15 \mathrm{sec}$, and then incubated for 2-3 $\mathrm{min}$ at room temperature. The sample was then centrifuged at $12,000 \mathrm{x} \mathrm{g}$ for $15 \mathrm{~min}$ at $4^{\circ} \mathrm{C}$. The aqueous phase was removed to a fresh tube, $0.5 \mathrm{ml}$ of $100 \%$ isopropanol per $1 \mathrm{ml}$ TRIzol was added to the aqueous phase, and the mixture was incubated at room temperature for $10 \mathrm{~min}$. Subsequently, the sample was centrifuged at $12,000 \mathrm{xg}$ for $10 \mathrm{~min}$ at $4^{\circ} \mathrm{C}$, the supernatant was removed, and the RNA pellet was washed with $75 \%$ (v/v) ethyl alcohol $(\mathrm{EtOH})$ and vortexed. This was followed by centrifugation at 7,500 $\mathrm{xg}$ for $5 \mathrm{~min}$ at $4^{\circ} \mathrm{C}$, removal of the supernatant and subsequent removal of the remaining EtOH by air drying for $5 \mathrm{~min}$. Finally, diethyl pyrocarbonate water (20-50 $\mathrm{ll}$ ) was added to resuspend the RNA pellets in the tube by passing the solution up and down several times through a pipette tip. The RNA concentrations were assessed by measuring absorbance at $260 \mathrm{~nm}$ using a NanoDrop ND-1000 spectrophotometer (Thermo Fisher Scientific, Inc.).

$R T-q P C R$. The expression of IncRNAs in ECC and adjacent non-cancerous tissues was measured by RT-qPCR using SYBR Premix Ex TaqÔ (Bioneer Corporation, Daejeon, Korea) and using the following cycling parameters: Initial denaturation at $94^{\circ} \mathrm{C}$ for $5 \mathrm{~min}$; followed by 40 cycles of $94^{\circ} \mathrm{C}$ for $30 \mathrm{sec} ; 60^{\circ} \mathrm{C}$ for $30 \mathrm{sec}$ and $72^{\circ} \mathrm{C}$ for $30 \mathrm{sec}$; and $72^{\circ} \mathrm{C}$ for $5 \mathrm{~min}$. Primers were designed by Sangon Biotech Co., Ltd. (Shanghai, China). GAPDH was used as a control. Experiments were performed in triplicate. The median in each triplicate was used to calculate relative lncRNA concentrations using the formula: $\Delta \mathrm{Cq}=\mathrm{Cq}_{\text {median }} \operatorname{lncRNAs-} \mathrm{Cq}_{\text {median }}$ GAPDH. Expression fold changes were calculated using the $2^{-\Delta \Delta C a}$ method (16). Primer sequences: ENST00000508732 forward, 5'-ACAGAGATAGCGGAAGGACA-3' and reverse, 5'-AATGGAGGACTGGAGGGATT-3'; ENST00000519319 forward, 5'-AAT GGCATG A AC CTG GGAGGCG-3' and reverse, 5'-GGCTTTGGGAAGTGCTTTGGAG-3'; UC022BVT forward, 5'-TGCTAAAGCATCAGAGAAGAG AAG-3' and reverse, 5'-GGACGTTCAACCTCATTCCC-3'; ENST00000438290 forward, 5'-GAGGGTTAAACCTGG AGAAGGG-3' and reverse, 5'-GCAAGAAAATGCGAGAAG CCT-3'; ENST00000593604 forward, 5'-CATGAGGACTGA GCGCATGA-3' and reverse, 5'-TGCAGTTCCTGTAGGTCA GA-3'; and GAPDH forward, 5'-AGAAGGCTGGGGCTC ATTTG-3' and reverse 5'-AGGGGCCATCCACAGTCTTC-3'.

Microarray analysis. The lncRNA and mRNA microarray data were analyzed for data summarization, normalization and quality control using the GeneSpring software version 13.0 (Agilent Technologies, Inc.). Differentially-expressed lncRNAs and mRNAs were determined based on $\mathrm{P}<0.05$, following Benjamini-Hochberg correction and a fold-change difference of $\geq 2.0$. The raw microarray data was $\log _{2}$-transformed and median-centered. The hierarchical clustering with average linkage was performed for genes and samples using CLUSTER 3.0 software (17). Finally, tree visualization was performed using Java Treeview (Stanford University School of Medicine, Stanford, CA, USA).

Construction of the lncRNA-mRNA co-expression network. The IncRNA-mRNA co-expression network was constructed based on association analysis between the differentially-expressed IncRNAs and mRNAs. For each pair of genes, Pearson's correlation coefficient was calculated and the significantly correlated pairs were selected to construct the network. LncRNAs and mRNAs with Pearson's correlation coefficients $>0.99$ were selected to draw the network.

Functional enrichment analysis. To investigate the potential functional roles of IncRNAs, functional enrichment analysis was performed at the gene ontology (GO; http://www.geneontology.org/) and Kyoto Encyclopedia of Genes and Genomes 
Table I. List of 10 differentially-expressed lncRNAs in ECC identified using a microarray screening in ECC and adjacent non-cancerous tissues (fold change, $>2.0 ; \mathrm{P}<0.05$ ).

\begin{tabular}{lrcccc}
\hline lncRNAs & Chr & Strand & Genomic coordinates & Expression \\
\hline ENST00000508732.2 & 15 & - & $95822513-95870329$ & Upregulated \\
TCONS_00004225 & 2 & - & $43199538-43228604$ & Downregulated & 21.486 \\
ENST00000438290.1 & 13 & + & $94712716-94716246$ & Downregulated & 7.547 \\
ENST00000423943.1 & 1 & + & $159931014-159948851$ & Downregulated & 6.386 \\
TCONS_00014813 & 8 & + & $102326509-102328921$ & Upregulated & 5.770 \\
ENST00000515485.1 & 4 & + & $165675216-165722606$ & Downregulated & 5.134 \\
ENST00000437097.1 & 9 & + & $128329858-128335302$ & Upregulated \\
TCONS_00008571 & 4 & - & $128015586-128017878$ & Downregulated & 4.079 \\
ENST00000606993.1 & 1 & + & $1104737-1105723$ & Downregulated & 4.043 \\
ENST00000550334.1 & 12 & - & $72255681-72271991$ & Downregulated & 4.042 \\
\hline
\end{tabular}

lncRNA, long non-coding RNA; Chr, chromosome; ECC, extrahepatic cholangiocarcinoma.

(KEGG; http://www.genome.jp/kegg/) levels using DAVID Bioinformatics Resources 6.7 (https://david.ncifcrf.gov/). $\mathrm{P}<0.05$ was considered to indicate statistically significant functional annotations.

Computational predictions of lncRNA targets. The regulatory roles of lncRNA on target genes were mediated by cis- and trans-acting mechanisms. The trans-prediction was conducted using BLAT tools to compare the full sequence of the lncRNA with the 3'UTR of its co-expression mRNAs, with the default parameter setting. For cis-acting lncRNAs, the regulatory RNAs were transcribed from the same locus as that which encodes the target gene and which was performed by their tight association (Pearson's association coefficient $>0.99$ ) to a group of expressed protein-coding genes. The lncRNA resided at genomic loci where a protein-coding gene and an lncRNA gene were within $10 \mathrm{~kb}$ of each other along the genome $(18,19)$; cis therefore refers to same-locus (not necessarily same-allele) regulatory mechanisms, which include antisense-mediated regulation by lncRNAs of protein-coding genes that are encoded in the same locus.

Statistical analysis. Statistical analysis was performed using SPSS version 18.0 software (SPSS, Inc., Chicago, IL, USA). Results are presented as the mean \pm standard deviation of three separate assays. Differences between groups were assessed using the two-tailed Student's t-test. $\mathrm{P}<0.05$ was considered to indicate a statistically significant difference.

\section{Results}

Identification of differentially-expressed lncRNAs and $m R N A s$ in ECC. Differential gene expression analysis in ECC and adjacent non-cancerous samples was performed to identify dysregulated lncRNAs and mRNAs in ECC. Among the 41,000 lncRNAs and 34,000 mRNAs transcripts accessed in the present microarray, it was identified that 268 lncRNAs and 459 mRNAs were differentially expressed (fold change $>2.0$ ) between tumor and adjacent non-cancerous samples. Among them, 78 lncRNAs and 66 mRNAs were upregulated ( $>2$-fold in ECC versus adjacent non-cancerous samples), and 190 lncRNAs and 393 mRNAs were downregulated in ECC samples. The 10 most differentially-expressed lncRNAs and mRNAs between ECC and adjacent non-cancerous tissue are listed in Table I. The data was $\log _{2}$-transformed and median-centered by genes using Adjust Data function of CLUSTER 3.0 software. Hierachial clustering analysis was then performed, and it was revealed that the expression profiles of differentially-expressed lncRNAs and mRNAs were able to distinguish ECC samples from normal tissue samples (Fig. 1A and B).

Functional analysis of differentially-expressed lncRNAs. To reveal the potential roles of IncRNAs in ECC, the association between lncRNAs and mRNAs was investigated, and a coding-non-coding gene co-expression (CNC) network was constructed by examining the association between the expression values of lncRNAs and those of the mRNAs. A total of 270 network nodes were associated with 5,788 network pairs of co-expressed lncRNAs and mRNA. The number of positively-associated pairs was greater than the number of negatively-associated pairs. The CNC network indicated that mRNAs may be associated with one or numerous lncRNAs. Similarly, lncRNAs may be associated with one or numerous mRNAs. XLOC_002797 had 38 neighbors, whereas collagen a-3 (type VI) mRNA had 29 neighbors. The CNC networkrevealed the inter-regulation of 1ncRNAs and mRNAs in ECC.

GO and KEGG function enrichment analysis was then performed for mRNAs co-expressed with lncRNAs to identify biological processes and signaling pathways affected by differentially-expressed lncRNAs. GO analysis revealed that the differentially-expressed mRNAs between ECC and adjacent non-cancerous tissue were significantly enriched in cellular response to ultraviolet-A rays, the sensory perception of pain, the creatinine metabolic process and protein processing. KEGG analysis indicated that the deregulated mRNAs between ECC and adjacent non-cancerous tissue were mainly involved in drug metabolism-cytochrome $\mathrm{P} 450$, nitrotoluene degradation, caffeine metabolism, the mitogen-activated 

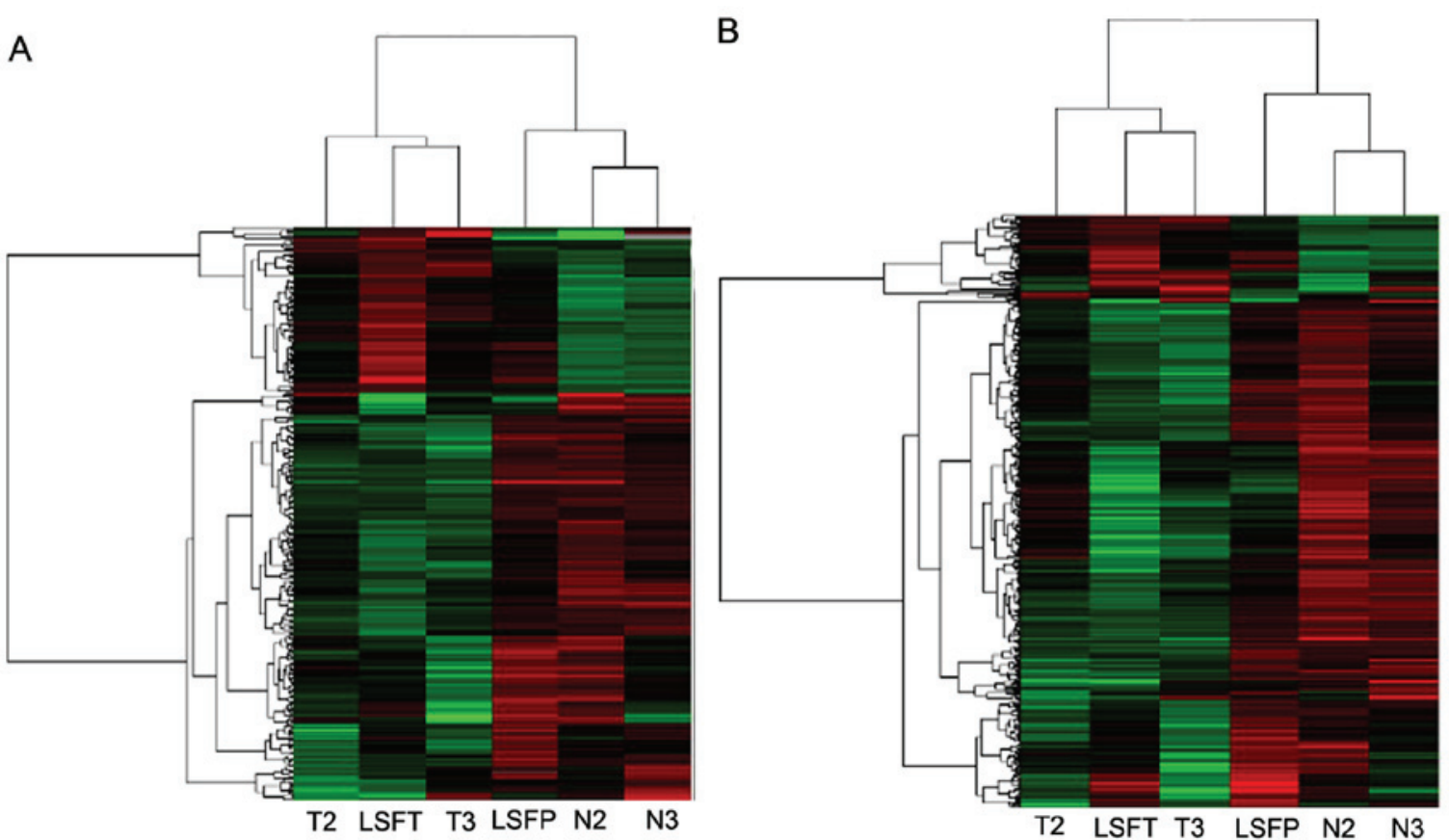

Figure 1. (A) Hierarchical clustering analysis of 268 differentially expressed long non-coding RNAs and (B) 459 differentially expressed mRNAs. Red and green colors indicate high and low expression, respectively. In the heat map, columns represent samples and rows represent each gene. T2, LSFT and T3 denote extrahepatic cholangiocarcinoma tissue samples; LSFP, N2 and N3 denote paired adjacent non-cancerous tissues.

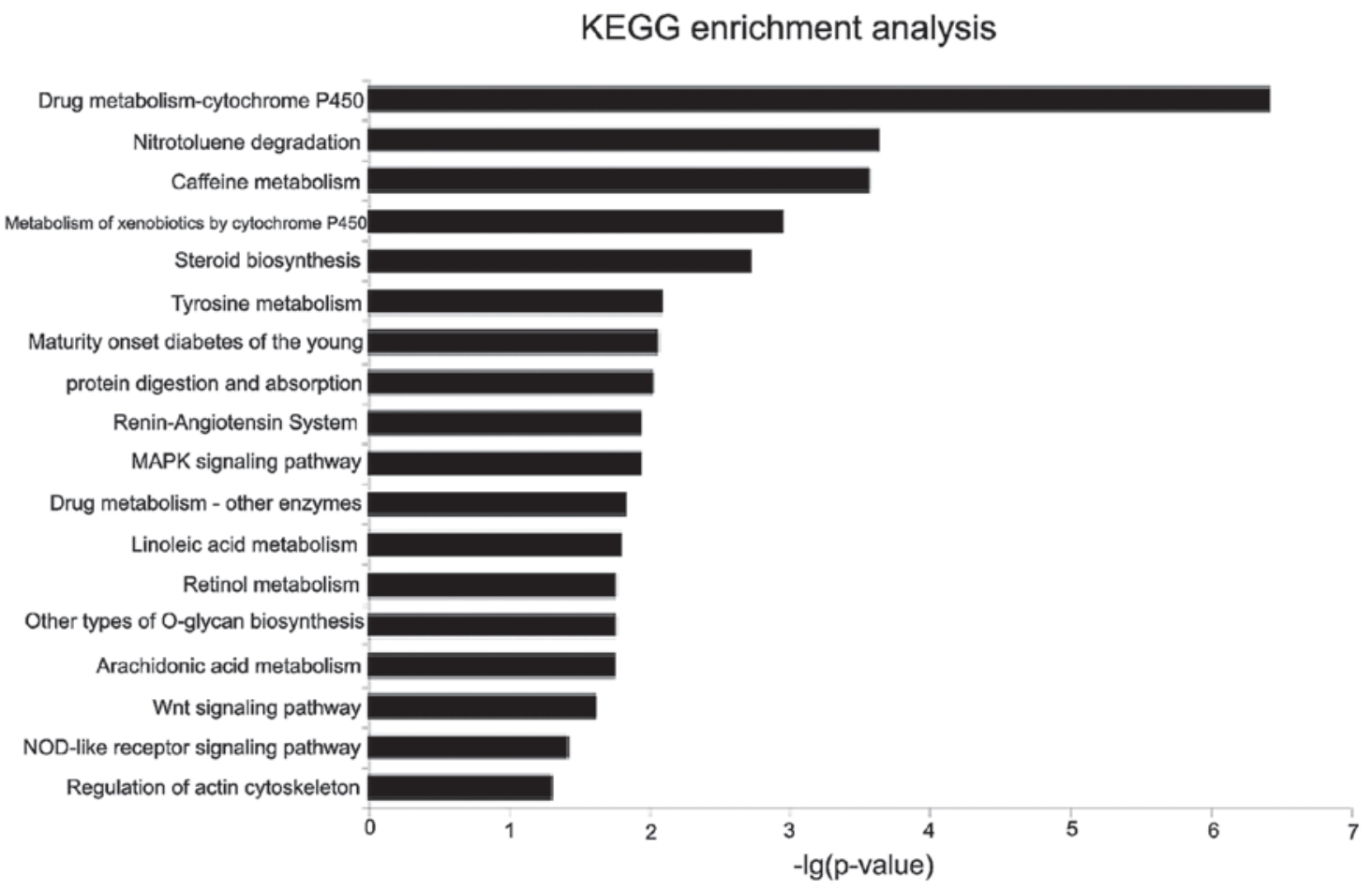

Figure 2. Significantly enriched KEGG pathways of mRNAs co-expressed with 1ncRNAs in the coding-non-coding gene co-expression network. KEGG, Kyoto Encyclopedia of Genes and Genomes; MAPK, mitogen-activated protein kinase; NOD, nucleotide oligomerization domain.

protein kinase (MAPK) signaling pathway, the peroxisome proliferator-activated receptor (PPAR) signaling pathway, protein digestion and absorption, the Wnt signaling pathway and the nucleotide oligomerization domain-like receptor signaling pathway $(\mathrm{P}<0.05$, following multiple testing correction; Fig. 2).
Validation of microarray results by $R T-q P C R$. In order to confirm aberrant lncRNA expression, 5 of the differentially-expressed lncRNAs in ECC were randomly selected for subsequent analysis to ensure the validity of microarray results using RT-qPCR in 42 pairs of ECC and adjacent non-cancerous tissues. The results revealed that 
Table II. Target prediction from lncRNAs to mRNAs.

\begin{tabular}{|c|c|c|c|c|c|}
\hline lncRNA & mRNA & Correlation & P-value & Cis-regulation & Trans-regulation \\
\hline p14589 & A_P186342 & 0.99929 & $2.387 \times 10^{-2}$ & & miRNA sequestration \\
\hline p29152 & A_P186342 & -0.99977 & $1.353 \times 10^{-2}$ & & miRNA sequestration \\
\hline p21976 & A_P0002916 & 0.99992 & $7.638 \times 10^{-3}$ & Sense & \\
\hline p17770 & A_P328023 & -0.99848 & $3.508 \times 10^{-2}$ & & miRNA sequestration \\
\hline p20598 & A_P132317 & -0.99908 & $2.722 \times 10^{-2}$ & & miRNA sequestration \\
\hline p6091 & A_P3414127 & 0.99851 & $3.469 \times 10^{-2}$ & & miRNA sequestration \\
\hline p9680 & A_P0001828 & 0.99999 & $6.390 \times 10^{-5}$ & Sense & \\
\hline p41956 & A_P3221253 & -0.99772 & $4.293 \times 10^{-2}$ & & miRNA sequestration \\
\hline
\end{tabular}

lncRNA, long non-coding RNA; miRNA, microRNA.

ENST00000508732, ENST00000519319 and UC022BVT were upregulated, while ENST00000438290 and ENST00000593604 were downregulated in ECC compared with adjacent non-cancerous tissue (Fig. 3). Overall, the present results demonstrated an association between RT-qPCR and microarray findings.

\section{Discussion}

lncRNAs are important regulators of gene expression during biological information processing and major cellular pathways, including proliferation, differentiation and apoptosis in living cells. Therefore, lncRNAs are involved in carcinogenesis or the antitumor effects of numerous human malignancies (20). However, thus far, knowledge about lncRNA expression in ECC is largely unknown.

A number of the differentially expressed lncRNAs and mRNAs that were identified in the present study are known to perform important roles in ECC. Differential expression of 268 lncRNA transcripts (defined as expression differences $>2$-fold) was observed. The expression was successfully validated for upregulated (ENST00000508732, ENST00000519319 and UC022BVT) and downregulated (ENST00000438290 and ENST00000593604) lncRNAs using qPCR in 42 pairs of ECC and adjacent non-cancerous frozen specimen. Thus, the specificity of the microarray results was confirmed.

If expression differences of IncRNAs are validated by independent researchers, these lncRNAs may represent diagnostic biomarkers or therapeutic targets in ECC. A previous study reported that 1 ncRNA may be a potential diagnostic and prognostic biomarker for intrahepatic cholangiocarcinoma (ICC), using lncRNA and mRNA microarrays, and also considered that the expression of lncRNA and mRNA may predict the survival of patients with ICC (21), although, as is commonlyunderstood, the embryogenesis, anatomy and biological behavior of ECC and ICC differ (22). Identification of diagnostic biomarkers or therapeutic targets in bodily fluids may assist to improve patient outcome and understanding of the molecular mechanismof cancer progression, for example,lncRNA $P C A 3$ in urine is used as a diagnostic biomarker for prostate cancer (23). CertainlncRNAs, including H19 (imprinted maternally-expressed transcript) and FENDRR (adjacent non-coding developmental regulatory

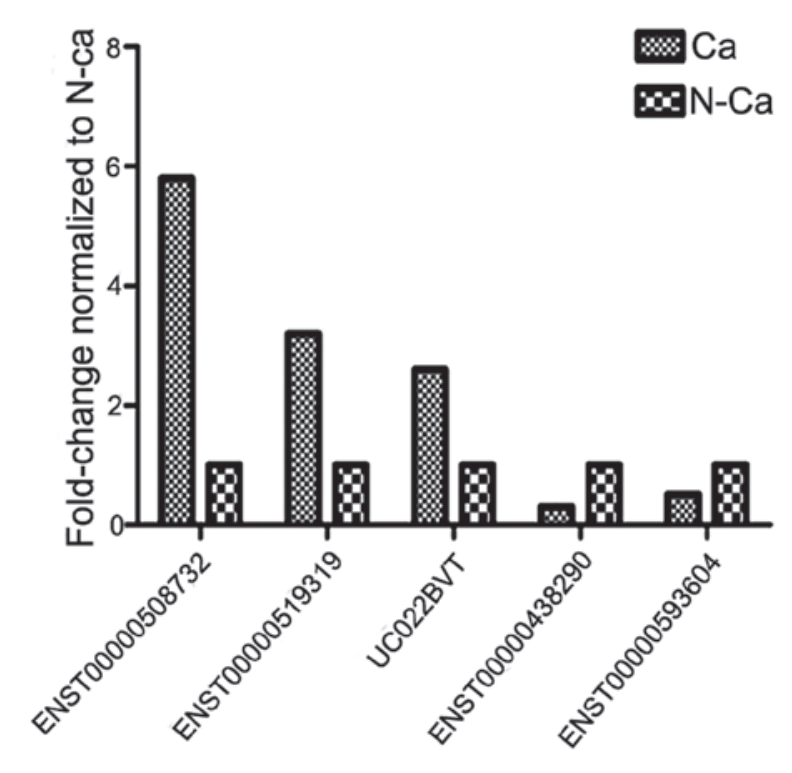

Figure 3. Selected lncRNA expression in microarray, and validation by reverse transcription-quantitative polymerase chain reaction.Ca, ECC tissues samples; N-Ca, paired adjacent non-cancerous tissues.

RNA), have been revealed to be differentially expressed in certain tumors $(24,25)$; this dysregulationwas also observedin ECC tissues by microarray detection in the present study. The role and mechanism of certain known lncRNAs in ECC require additional investigations.

However, little is known on the function of lncRNAs and how to research them. Therefore, microarrays of lncRNAs and mRNAs may assist to elucidate this through certain bioinformatics methods, including CNC network and target gene predictions (cis and trans). The present results may provide clues for additional basic studies (Table II). The theory of target gene predictions may reveal that lncRNA functions via lncRNA-mRNA-protein interactions (26). The majority of these proteins code genes that function in the splicing, binding, transport, localization, transcription, translation and processing of RNA, according to GO function prediction.

It has also been reported that lncRNAs may act as a microRNA sponge by binding specific microRNAs and thereby disrupting microRNA regulation of mRNA 3'UTRs (27). In the present study, 39 IncRNAswere predicted 
to target mRNAs by comparingsequences of lncRNAs with the 3'UTR of mRNAs. The miRTarget2 algorithm (28), starBase(http://starbase.sysu.edu.cn/) (29) and miRcode (www.mircode.org/) (30) were used to predict miRNA seeds within the validated lncRNA transcripts, which may assist in constructing the IncRNA-miRNA-mRNA axis. Prensner et al showed that prostate cancer-associated transcript 1 is able to abrogate the downregulation of cMyc by downregulating the expression of miR-34a in prostate cancer (4).

KEGG analysis has revealed that drug metabolism-cytochrome P450 (31), nitrotoluene degradation and caffeine metabolism induced by $\mathrm{N}$-acetyltransferase (NAT) 1 and NAT2 (32) have significant association with the genesis and development of ECC, with the exception of classical proliferation and apoptotic pathways (such as the PPAR and MAPK pathways).

In summary, the present study revealed that dysregulation of $\sim 4 \%$ of the lncRNA transcripts occurs in ECC, and altered lncRNA expression may modulate fundamental cellular processes. IncRNA profiles were able to accurately distinguish between ECC and adjacent non-cancer tissue. Thus, lncRNAs may be used as biomarkers and therapeutic targets for patients with ECC.

\section{References}

1. Khan SA, Emadossadaty S, Ladep NG, Thomas HC, Elliott P, Taylor-Robinson SD and Toledano MB: Rising trends in cholangiocarcinoma: Is the ICD classification system misleading us? J Hepatol 56: 848-54, 2012.

2. DeOliveira ML, Cunningham SC, Cameron JL, Kamangar F, Winter JM, Lillemoe KD, Choti MA, Yeo CJ and Schulick RD: Cholangiocarcinoma: Thirty-one-year experience with 564 patients at a single institution. Ann Surg 245: 755-762, 2007.

3. van der Gaag NA, Kloek JJ, de Bakker JK, Musters B, Geskus RB, Busch OR, Bosma A, Gouma DJ and van Gulik TM: Survival analysis and prognostic nomogram for patients undergoing resection of extrahepatic cholangiocarcinoma. Ann Oncol 23: 2642-2649, 2012.

4. Sun M, Jin FY, Xia R, Kong R, Li JH, Xu TP, Liu YW, Zhang EB, Liu XH and De W: Decreased expression of long noncoding RNA GAS5 indicates a poor prognosis and promotes cell proliferation in gastric cancer. BMC Cancer 14: 319, 2014.

5. Prensner JR, Chen W, Han S, Iyer MK, Cao Q, Kothari V, Evans JR, Knudsen KE, Paulsen MT, Ljungman M, et al: The long non-coding RNA PCAT-1 promotes prostate cancer cell proliferation through cMyc. Neoplasia 16: 900-908, 2014.

6. Hu Y, Chen HY, Yu CY, Xu J, Wang JL, Qian J, Zhang X and Fang JY: A long non-coding RNA signature to improve prognosis prediction of colorectal cancer. Oncotarget 5: 2230-2242, 2014.

7. Sun J, Chen X, Wang Z, Guo M, Shi H, Wang X, Cheng L and Zhou M: A potential prognostic long non-coding RNA signature to predict metastasis-free survival of breast cancer patients. Sci Rep 5: 16553, 2015.

8. Zhou M, Guo M, He D, Wang X, Cui Y, Yang H, Hao D and Sun J: A potential signature of eight long non-coding RNAs predicts survival in patients with non-small cell lung cancer. J Transl Med 13: 231, 2015.

9. Zhou M, Wang X, Shi H, Cheng L, Wang Z, Zhao H, Yang L and Sun J: Characterization of long non-coding RNA-associated ceRNA network to reveal potential prognostic lncRNA biomarkers in human ovarian cancer. Oncotarget 7: 12598-12611, 2016.

10. Zhou M, Zhao H, Wang Z, Cheng L, Yang L, Shi H, Yang H and Sun J: Identification and validation of potential prognostic lncRNA biomarkers for predicting survival in patients with multiple myeloma. J Exp Clin Cancer Res 34: 102, 2015.

11. Cheng N, Li X, Zhao C, Ren S, Chen X, Cai W, Zhao M, Zhang Y, Li J, Wang Q and Zhou C: Microarray expression profile of long non-coding RNAs in EGFR-TKIs resistance of human non-small cell lung cancer. Oncol Rep 33: 833-839, 2015.
12. Li C, Liang G, Yao W, Sui J, Shen X, Zhang Y, Ma S, Ye Y, Zhang Z, Zhang W, et al: Differential expression profiles of long non-coding RNAs reveal potential biomarkers for identification of human gastric cancer. Oncol Rep 35: 1529-1540, 2016.

13. Luo J, Xu L, Jiang Y, Zhuo D, Zhang S, Wu L, Xu H and Huang Y: Expression profile of long non-coding RNAs in colorectal cancer: A microarray analysis. Oncol Rep 35: 2035-2044, 2016.

14. Qiu JJ, Ye LC, Ding JX, Feng WW, Jin HY, Zhang Y, Li Q and Hua KQ: Expression and clinical significance of estrogen-regulated long non-coding RNAs in estrogen receptor $\alpha$-positive ovarian cancer progression. Oncol Rep 31: 1613-1622, 2014.

15. Ørom UA, Derrien T, Beringer M, Gumireddy K, Gardini A, Bussotti G, Lai F, Zytnicki M, Notredame C, Huang Q, et al: Long noncoding RNAs with enhancer-like function in human cells. Cell 143: 46-58, 2010.

16. Livak KJ and Schmittgen TD: Analysis of relative gene expression data using real-time quantitative PCR and the 2(-Delta Delta C(T)) method. Methods 25: 402-408, 2001.

17. Eisen MB, Spellman PT, Brown PO and Botstein D: Cluster analysis and display of genome-wide expression patterns. Proc Natl Acad Sci USA 95: 14863-14868, 1998.

18. Jia H, Osak M, Bogu GK, Stanton LW, Johnson R and Lipovich L: Genome-wide computational identification and manual annotation of human long noncoding RNA genes. RNA 16: 1478-1487, 2010.

19. Sun J, Zhou M, Mao ZT, Hao DP, Wang ZZ and Li CX: Systematic analysis of genomic organization and structure of long non-coding RNAs in the human genome. FEBS Lett 587: 976-982, 2013.

20. Martens-Uzunova ES, Bottcher R, Croce CM, Jenster G, Visakorpi T and Calin GA: Long noncoding RNA in prostate, bladder, and kidney cancer. Eur Urol 65: 1140-1151, 2014.

21. Wang J, Xie H, Ling Q, Lu D, Lv Z, Zhuang R, Liu Z, Wei X, Zhou L, Xu X and Zheng S: Coding-noncoding gene expression in intrahepatic cholangiocarcinoma. Transl Res 168: 107-121, 2016.

22. ICD-O-Cs (International Classification of DiseasesOncology-Czech edition). Cesk Patol 19: 228-250, 1983 (In Czech).

23. Bradley LA, Palomaki GE, Gutman S, Samson D and Aronson N: Comparative effectiveness review: Prostate cancer antigen 3 testing for the diagnosis and management of prostate cancer. J Urol 190: 389-398, 2013.

24. Raveh E, Matouk IJ, Gilon M and Hochberg A: The H19 Long non-coding RNA in cancer initiation, progression and metastasis-a proposed unifying theory. Mol Cancer 14: 184, 2015.

25. Xu TP, Huang MD, Xia R, Liu XX, Sun M, Yin L, Chen WM, Han L, Zhang EB, Kong R, et al: Decreased expression of the long non-coding RNA FENDRR is associated with poor prognosis in gastric cancer and FENDRR regulates gastric cancer cell metastasis by affecting fibronectin 1 expression. J Hematol Oncol 7: 63, 2014.

26. Zhu J, Fu H, Wu Y and Zheng X: Function of lncRNAs and approaches to IncRNA-protein interactions. Sci China Life Sci 56: 876-885, 2013.

27. Salmena L, Poliseno L, Tay Y, Kats L and Pandolfi PP: A ceRNA hypothesis: The Rosetta stone of a hidden RNA language? Cell 146: 353-358, 2011

28. Wang $X$ and El Naqa IM: Prediction of both conserved and nonconserved microRNA targets in animals. Bioinformatics 24: 325-332, 2008

29. Li JH, Liu S, Zhou H, Qu LH and Yang JH: Decoding miRNA-ceRNA, miRNA-ncRNA and protein-RNA interaction networks from large-scale CLIP-Seq data. Nucleic Acids Res 42 (Database Issue): D92-D97, 2014.

30. Jeggari A, Marks DS and Larsson E: miRcode: A map of putative microRNA target sites in the long non-coding transcriptome. Bioinformatics 28: 2062-2063, 2012.

31. Yongvanit P, Phanomsri E, Namwat N, Kampan J, Tassaneeyakul W, Loilome W, Puapairoj A and Khuntikeo N: Hepatic cytochrome P450 2A6 and 2E1 status in peri-tumor tissues of patients with Opisthorchis viverrini-associated cholangiocarcinoma. Parasitol Int 61: 162-166, 2012.

32. Prawan A, Kukongviriyapan V, Tassaneeyakul W, Pairojkul C and Bhudhisawasdi V: Association between genetic polymorphisms of CYP1A2, arylamine $\mathrm{N}$-acetyltransferase 1 and 2 and susceptibility to cholangiocarcinoma. Eur J Cancer Prev 14: 245-250, 2005. 